\title{
MicroRNA-200b relieves LPS-induced inflammatory injury by targeting FUT4 in knee articular chondrocytes in vitro
}

\author{
YINTAI LI $^{1}$, SUIZHUAN WEI ${ }^{2}$ and ZHONGPING ZHANG ${ }^{3}$ \\ Departments of ${ }^{1}$ Rehabilitation and ${ }^{2}$ Orthopedics, Baoji Traditional Chinese Medicine Hospital, Baoji, Shaanxi 721000; \\ ${ }^{3}$ Department of Orthopedics, Yan'an People's Hospital, Yan'an, Shaanxi 716000, P.R. China
}

Received September 30, 2019; Accepted September 29, 2020

DOI: $10.3892 /$ etm.2021.9838

\begin{abstract}
Osteoarthritis (OA), characterized by the degeneration of articular cartilage, is a major problem in aging populations, and cartilage chondrocytes have been indicated to serve a curial role in the progression of OA. MicroRNA-200b-3p (miR-200b) was preliminarily identified to participate in OA. However, its role and mechanism of action in injured chondrocytes in OA remain unclear to date. In the present study, lipopolysaccharide (LPS)-treated cells isolated from normal knee articular cartilage were used to mimic inflammatory injury of OA chondrocytes. Cell viability, apoptosis and inflammatory responses were detected using Cell Counting Kit-8, flow cytometry and enzyme-linked immunosorbent assay, respectively. The expression levels of miR-200b and fucosyltransferase-4 (FUT4) were measured by reverse transcription-quantitative PCR and western blotting. The association between miR-200b and FUT4 was verified using TargetScan software, dual-luciferase reporter assay and RNA immunoprecipitation. The results indicated that LPS treatment decreased cell viability of primary chondrocytes, and increased apoptosis rate and production of IL-1 $\beta$, IL-6 and TNF- $\alpha$. The expression level of miR-200b was downregulated, and that of FUT4 was upregulated in OA cartilage tissues and LPS-treated normal chondrocytes compared with normal cartilage tissues and chondrocytes. Overexpression of miR-200b via transfection with miR-200b mimic inhibited the apoptosis rate and reduced the levels of IL-1 $\beta$, IL- 6 and TNF- $\alpha$ in LPS-stimulated chondrocytes. However, the suppressive effect of miR-200b overexpression on the LPS-induced
\end{abstract}

Correspondence to: Dr Zhongping Zhang, Department of Orthopedics, Yan'an People's Hospital, 57 Qilipu Street, Yan'an, Shaanxi 716000, P.R. China

E-mail: i3007093shaorang@163.com

Abbreviations: OA, osteoarthritis; LPS, lipopolysaccharide; CCK-8, Cell Counting Kit-8; RT-qPCR, reverse transcription-quantitative PCR; RIP, RNA immunoprecipitation

Key words: microRNA-200b, LPS, chondrocytes, fucosyltransferase-4, $\mathrm{OA}$, inflammatory injury inflammatory injury in chondrocytes was reversed by the restoration of FUT4 levels. Notably, FUT4 was indicated to be a downstream target of miR-200b and was negatively regulated by miR-200b. Taken together, the results of the current study indicated that miR-200b protected chondrocytes from LPS-induced inflammatory injury in vitro by targeting FUT4. These findings revealed the miR-200b/FUT4 axis as a potential candidate to target the degeneration of cartilages, thereby inhibiting the progression of OA.

\section{Introduction}

Osteoarthritis (OA) is the most prevalent joint disease, and has been considered to be a type of non-inflammatory arthritis for a number of years (1). The characteristics of OA include pain, tenderness, limited movement, crepitus and different levels of inflammation without systemic effects (2). Due to the close association with age, OA has become one of the major problems in elderly individuals (3). Articular cartilage degradation is one of the principal pathological alterations in OA, and the fate of articular cartilage is determined by articular chondrocytes (4). Mechanistically, the imbalance in extracellular matrix (ECM) synthesis and degradation results in injuries in articular cartilage, including inflammatory injury. Moreover, apoptosis of chondrocytes has been suggested to serve an essential role in the pathogenesis of OA (5). Therefore, chondrocytes, as the only cell type in articular cartilage (6), have been most commonly used in OA research to elucidate the pathogenesis of this condition.

Increasing evidence has suggested that non-coding RNAs participate in OA (7), and microRNAs (miRNAs/miRs) are one class of non-coding RNAs with about 22 nucleotides (8). Recently, dysregulation of miRNAs has also been indicated to be associated with the maintenance of cartilage homeostasis and inflammatory response during the progression of OA (9), including miR-320, miR-21 and miR-140 (10-12). miR-200b-3p (miR-200b) has been revealed to be an important regulator of cell proliferation and motility in various types of cancer, such as cervical cancer and breast cancer (13-15). However, although the regulatory mechanism of miR-200b has been preliminarily identified in cartilage cells from patients with OA, its potential role in OA has not been widely studied (16).

As key membrane-bound proteins in the surface of cells residing in the endoplasmic reticulum and Golgi (17), 
fucosyltransferases (FUTs) have been reported to participate in miscellaneous biological processes, including inflammation $(15,18)$. Accumulating evidence has indicated that FUTs may regulate the development of rheumatoid arthritis (RA) and juvenile idiopathic arthritis $(19,20)$, whereas the effect of FUTs in OA remains not fully understood. FUT4 has been indicated to mediate OA progression following miR-26a/b targeting (21). The present study examined the association between FUT4 and miR-200b in injured chondrocytes in OA.

Lipopolysaccharide (LPS), also known as endotoxin, can induce inflammatory response both in vitro and in vivo (22). Numerous inflammatory cytokines have been indicated to be highly expressed after LPS treatment, such as interleukin IL-1 $\beta$, IL-6, IL-8 and TNF- $\alpha$ (23). Therefore, LPS-stimulated cell models have been widely used to identify novel therapeutic targets for the treatment of OA (23-25). Moreover, miRNAs have been revealed to be implicated in LPS-induced articular chondrocyte injury in OA $(4,24,25)$.

The present study aimed to investigate the expression level of miR-200b in cartilage tissues from patients with OA and its role in an LPS-induced chondrocyte OA model in vitro. A mechanistic study was also performed to explore the association between miR-200b and FUT4 in modulating inflammatory injury in LPS-treated knee articular chondrocytes.

\section{Materials and methods}

Patients and tissue specimens. Normal cartilage specimens were obtained from 14 patients with emergency traumatic amputation ( 8 males and 6 females; average age, 34.6 years) and 14 patients with OA ( 5 males and 9 females; average age, 45.3 years) between Jan, 2014 and Dec. 2016 at the Yan'an People's Hospital (Yan'an, China). The average body height and weight were $165.6 \mathrm{~cm}$ and $65.4 \mathrm{~kg}$ in normal patients and $162.3 \mathrm{~cm}$ and $62.7 \mathrm{~kg}$ in patients with OA. All normal patients with arthritis, including RA, OA and septic arthritis, were excluded from the present study. Among the patients with OA, patients were excluded if they received orthopedic surgery before, or suffered a fracture, septic arthritis or other serious diseases. The pathological diagnosis was confirmed by two professor pathologists, and the diagnostic criteria of knee OA used were in accordance with the International Classification of Diseases, Tenth Revision, Clinical Modification (code M17) (26). The samples were isolated form the knee articular cartilage and subsequently placed into liquid nitrogen at $-73^{\circ} \mathrm{C}$. Written informed consent was obtained from each patient and the study was approved by the Ethics Committee of Yan'an People's Hospital. Moreover, the present study involving human subjects, human material or human data was performed in accordance with the Declaration of Helsinki.

Cell culture and LPS stimulation. Under aseptic conditions, normal cartilage tissues and OA cartilages were removed from fibrous connective tissues and cut into small pieces. After washing with PBS containing $100 \mathrm{U} / \mathrm{ml}$ penicillin and $100 \mu \mathrm{g} / \mathrm{ml}$ streptomycin solution (Gibco; Thermo Fisher Scientific, Inc.), the cartilage tissues were subjected to sequential digestion with $0.25 \%$ trypsin (Invitrogen; Thermo Fisher Scientific, Inc.) for $30 \mathrm{~min}$ at $37^{\circ} \mathrm{C}$ and subsequently with
$0.2 \%$ collagenase type II (EMD Millipore) in DMEM (Gibco; Thermo Fisher Scientific, Inc.) for $10 \mathrm{~h}$ at $37^{\circ} \mathrm{C}$. Following filtration with filters (100 and $40 \mu \mathrm{m}$; JingAn Biological), the cells in medium were centrifuged at $400 \mathrm{x} g$ for $15 \mathrm{~min}$ at room temperature, and subsequently resuspended in fresh culture media supplemented with $10 \%$ FBS (Gibco; Thermo Fisher Scientific, Inc.). The cartilage cells and HEK 293T (293T) (cat. no. CRL-3216; American Type Culture Collection) cells were cultured in DMEM (Gibco; Thermo Fisher Scientific, Inc.) containing 10\% FBS (Gibco; Thermo Fisher Scientific, Inc.) in humidified air with $5 \%(\mathrm{v} / \mathrm{v}) \mathrm{CO}_{2}$ at $37^{\circ} \mathrm{C}$. The first passage chondrocytes were obtained after cell cultivation for 10 days.

All experiments were performed on cells between the first and third passage stimulated with LPS (MilliporeSigma), and the cells were treated with $0.25 \%$ trypsin (Invitrogen; Thermo Fisher Scientific, Inc.) for $1 \mathrm{~min}$ at $37^{\circ} \mathrm{C}$ between passages. LPS was dissolved in ultrapure water at a stock concentration of $5 \mathrm{mg} / \mathrm{ml}$ according to the manufacturer's instructions. For LPS stimulation, chondrocytes were incubated in serum-free DMEM containing LPS at 1,5 and $10 \mu \mathrm{g} / \mathrm{ml}$ for $48 \mathrm{~h}$ at $37^{\circ} \mathrm{C}$. The blank group was incubated in serum-free DMEM without LPS.

Cell Counting Kit (CCK)-8 assay. The viability of the chondrocytes was determined using CCK-8 kit (Dojindo Molecular Technologies, Inc.). In brief, the chondrocytes were seeded into 96-well plates (Corning, Inc.) at a density of $1 \times 10^{4}$ cells/well for $24 \mathrm{~h}$, and subsequently treated with $0,1,5$ and $10 \mu \mathrm{g} / \mathrm{ml} \mathrm{LPS}$ for $48 \mathrm{~h}$ as aforementioned. The cells were cultured with $20 \mu \mathrm{l}$ CCK-8 solution ( $5 \mathrm{~g} / \mathrm{l})$ in PBS for another $2 \mathrm{~h}$, and the optical density at $450 \mathrm{~nm}$ was measured on a microplate reader. Each group was assayed in triplicate.

Flow cytometry. For cell apoptosis analysis, LPS-treated chondrocytes were seeded into 6-well plates (Corning, Inc.) at a density of $1 \times 10^{5}$ cells/well for $24 \mathrm{~h}$ and were subsequently analyzed with flow cytometry using an Annexin V-FITC apoptosis detection kit (Beyotime Institute of Biotechnology). The apoptotic cells were labelled according to the manufacturer's protocol. In brief, the adherent and floating cells were harvested and washed twice with PBS three times. Subsequently, $100 \mu 1$ cells of each group were stained in a binding buffer containing Annexin V-FITC and PI for $30 \mathrm{~min}$ at $4^{\circ} \mathrm{C}$ in the dark. Fluorescence was analyzed with CytoFLEX LX flow cytometer (Beckman Coulter, Inc.) using CytExpert software (version 2.0; Beckman Coulter, Inc.). The blank group was treated with $0 \mu \mathrm{g} / \mathrm{ml}$ LPS, and the control groups were transfected with miR-NC mimic or co-transfected with miR-200b mimic and pcDNA. For cell surface marker detection, cartilage cells on passage 1 were collected by $0.25 \%$ trypsin (Invitrogen; Thermo Fisher Scientific, Inc.) for $1 \mathrm{~min}$ at $37^{\circ} \mathrm{C}$, and washed with PBS three times; $1 \times 10^{7}$ cells were resuspended in the binding buffer, followed by incubation with monoclonal antibodies against CD44 (cat. no. AF0105; 1:100; Beyotime Institute of Biotechnology) and CD151 (cat. no. FAB1884P; 1:100; R\&D Systems China Co., Ltd.) conjugated to FITC or phycoerythrin for $30 \mathrm{~min}$ at room temperature in the dark. After washing with PBS once, these cells were analyzed with a FACScan flow cytometer (Becton-Dickinson and Company), using CellQuest software 
(version 5.1; Becton-Dickinson and Company). The results were obtained from three independent experiments.

ELISA. ELISA was performed to measure the concentration of IL-1 $\beta$, IL- 6 and TNF- $\alpha$ that were released in the culture supernatants. Chondrocytes without and with transfection were seeded into 24-well plates (Corning, Inc.) for $24 \mathrm{~h}$. After LPS $(0,1,5$ and $10 \mu \mathrm{g} / \mathrm{ml})$ stimulation, the culture supernatants were collected to measure the inflammatory factor levels according to the manufacturer's instructions of each kit. The ELISA kits used were as follows: Human IL-1 $\beta$ (cat. no. ab100562), human IL-6 (cat. no. ab46027) and human TNF- $\alpha$ ELISA kit (cat. no. ab46087), all from Abcam. Three independent experiments in triplicate were performed.

Reverse transcription-quantitative PCR (RT-qPCR). Total RNA from OA cartilages and LPS-treated chondrocytes was isolated using TRIzol ${ }^{\circledR}$ reagent (Thermo Fisher Scientific, Inc.). Briefly, $300 \mathrm{ng}$ total RNA was used to synthesize cDNA at $42^{\circ} \mathrm{C}$ for $15 \mathrm{~min}$ using All-in-one MasterMix (Applied Biological Materials Inc.) for mRNA and Bestar ${ }^{\mathrm{TM}} \mathrm{qPCR}$ RT kit for miRNA (DBI Bioscience). The amplification of cDNA was performed using SYBR ${ }^{\circledR}$ Premix Ex Taq Master Mix (Takara Bio, Inc.) or Bestar ${ }^{\circledR}$ SYBR Green qPCR Master Mix (DBI Bioscience) on the ABI PRISM 7500 Real-time PCR System (Applied Biosystems; Thermo Fisher Scientific, Inc.). The thermocycling conditions were 40 cycles of $95^{\circ} \mathrm{C}$ for $15 \mathrm{sec}, 60^{\circ} \mathrm{C}$ for $60 \mathrm{sec}$ and $95^{\circ} \mathrm{C}$ for $15 \mathrm{sec}$. RNA expression was calculated using the $2^{-\Delta \Delta \mathrm{Cq}}$ method (27). All primers were synthesized by Shanghai GenePharma Co., Ltd. and are listed in Table I. All experiments were performed at least in triplicate. The data are presented as fold change relative to control levels. GAPDH (for mRNA) or U6 (for miRNA) were used for normalization.

Cell transfection. The primary chondrocyte were seeded into six-well plates (Corning, Inc.) at a density of $5 \times 10^{4}$ cells/well for $24 \mathrm{~h}$ prior to transfection, and 293T cells were pre-seeded in 24 -well plate at a density of $5 \times 10^{3}$ cells/well. For overexpression purposes, the coding domain sequence of FUT4 was inserted into pcDNA3.1 vector (Invitrogen; Thermo Fisher Scientific, Inc.), and equal amount of empty vector was used as negative control. miR-200b mimic and its negative control miR-NC mimic were purchased from Shanghai GenePharma Co., Ltd.. For knockdown purposes, anti-miR-200b and anti-miR-NC were provided by Shanghai GenePharma Co., Ltd.. Cell transfection was performed using Lipofectamine ${ }^{\circledR}$ 3000 reagent (Invitrogen; Thermo Fisher Scientific, Inc.) in serum-free DMEM at $37^{\circ} \mathrm{C}$ for $6 \mathrm{~h}$, and the culture medium was replaced with complete cell culture DMEM medium for $48 \mathrm{~h}$ prior to further analysis. Briefly, $2 \mu \mathrm{g}$ vectors and $30 \mathrm{nM}$ oligonucleotides were transfected into cells in six-well plate, and $20 \mathrm{ng}$ vectors and $20 \mathrm{nM}$ oligonucleotides were transfected in a 24-well plate. After transfection for $48 \mathrm{~h}$, chondrocytes were treated with 0 or $10 \mu \mathrm{g} / \mathrm{ml}$ LPS at $37^{\circ} \mathrm{C}$ for another $48 \mathrm{~h}$.

Dual-luciferase reporter assay. Prediction of potential targets was performed using TargetScan software (version 7.2; http://www.targetscan.org/vert_72/). The putative binding sites of hsa-miR-200b in the 3 '-untranslated region
Table I. Sequences of primers used in reverse transcriptionquantitative PCR.

\begin{tabular}{ll} 
Gene & \multicolumn{1}{c}{ Primer sequence (5'-3') } \\
\hline miR-200b & F: GCTGCTGAATTCCATCTAATTTCCAAAAG \\
& R: TATTATGGATCCGCCCCCAGGGCAATGGG \\
FUT4 & F: TCCTACGGAGAGGCTCAG \\
& R: TCCTCGTAGTCCAACACG \\
U6 & F: AACGCTTCACGAATTTGCGT \\
& R: CTCGCTTCGGCAGCACA \\
GAPDH & F: GTCAACGGATTTGGTCTGTATT \\
& R: AGTCTTCTGGGTGGCAGTGAT \\
\hline
\end{tabular}

miR-200b, miRNA-200b-3p; FUT4, fucosyltransferase 4; U6, U6 small nuclear RNA; F, forward; R, reverse.

of FUT4 (FUT4 3'-UTR) were mutated (FUT4-MUT). Subsequently, the wild-type FUT4 3'-UTR (FUT4-WT) and FUT4-MUT were cloned into pMIR-Luciferase Reporter Vector (Invitrogen; Thermo Fisher Scientific, Inc.). 293T cells (cat. no. CRL-3216; American Type Culture Collection; $1 \times 10^{4}$ cells/well) in the logarithmic growth phase were plated in 24-well plates (Corning, Inc.), followed by co-transfection with $20 \mathrm{nM}$ miR-200b/NC mimic and $20 \mathrm{ng}$ FUT4-WT/MUT using Lipofectamine ${ }^{\circledR} 3000$ reagent (Invitrogen; Thermo Fisher Scientific, Inc.). After transfection for $48 \mathrm{~h}$, the cells were collected to measure the relative luciferase activity using the Dual-Luciferase ${ }^{\circledR}$ Reporter Assay System (Promega Corporation) with comparison with Renilla luciferase. All transfections were performed in triplicate.

RNA immunoprecipitation (RIP). Magna RIP ${ }^{\mathrm{TM}}$ RNA-binding protein immunoprecipitation kit (EMD Millipore; cat. no. 17-700) was used for RIP assay in chondrocyte extracts after transfection with miR-200b/NC mimic. Immunoprecipitation of mRNAs were bound to $5 \mu \mathrm{l}$ argonaute-2 (Ago2; cat. no. 03-110; EMD Millipore) or IgG (control; 03-198; EMD Millipore) antibody. The immunoprecipitated mRNAs were isolated using TRIzol reagent (Thermo Fisher Scientific, Inc.) and FUT4 mRNA expression was measured via RT-qPCR. All procedures were performed according to the manufacturer's protocol.

Western blotting. Total protein from LPS-treated chondrocytes was extracted using RIPA lysis buffer (Beyotime Institute of Biotechnology), and protein concentration was determined using a BCA Protein Assay kit (Pierce; Thermo Fisher Scientific, Inc.). A total of $20 \mu \mathrm{g}$ of proteins/lane were resolved on SDS-PAGE (12\% gel), and transferred onto PVDF membranes (EMD Millipore). The membranes were blocked with $5 \%$ nonfat milk for $1 \mathrm{~h}$ at $25^{\circ} \mathrm{C}$, and subsequently incubated with primary antibodies overnight at $4^{\circ} \mathrm{C}$. The following primary antibodies were supplied by Abcam: Anti-FUT4 (1:500; cat. no. ab181461) and anti- $\beta$-actin $(1: 1,000$; cat. no. ab8227). After incubation with HRP-conjugated secondary antibodies against mouse (cat. no. A0216; 1:1,000; Beyotime 
Institute of Biotechnology) or rabbit (cat. no. A0208; 1:1,000; Beyotime Institute of Biotechnology) at room temperature for $1.5 \mathrm{~h}$, the bands were visualized using an ECL kit (EMD Millipore) according to standard protocols. $\beta$-actin was used as an internal standard to normalize protein levels analyzed on Image-Pro Plus software (version 6.0; Media Cybernetics, Inc.). Western blotting of the same proteins was performed in triplicate.

Statistical analysis. Data are presented as the mean \pm SD from three independent experiments. Statistical significance was determined using independent Student's t-test for comparisons between two groups and one-way ANOVA followed by Tukey's post hoc test for comparisons among multiple groups. Data analysis was performed using GraphPad Prism v6.0 (GraphPad Software Inc.). $\mathrm{P}<0.05$ was considered to indicate a statistically significant difference.

\section{Results}

LPS-induced inflammatory injury in knee articular chondrocytes in vitro. In the present study, patients with OA and control patients were recruited for knee cartilage tissue isolation. OA cartilages were used for the separation of OA chondrocytes (Fig. S1), while normal chondrocytes were isolated from normal cartilages, followed by LPS treatment for the establishment of the OA model. Firstly, the cultured cells from normal cartilages were analyzed using surface markers of chondrocytes (CD44 and CD151) via flow cytometry. The results indicated that $83.2 \%$ of the cells were $\mathrm{CD} 44^{+} / \mathrm{CD} 151^{+}$ (Fig. S2), suggesting that the majority of the cells were chondrocytes. Subsequently, a cell model of LPS-induced chondrocyte injury in vitro was established and verified. Chondrocytes were exposed to LPS, and cell behaviors were measured. As presented in Fig. 1A, 5 and $10 \mu \mathrm{g} / \mathrm{ml}$ LPS induced a significant decrease in cell viability compared with control cells, while the apoptosis rate was increased from 6.9 to $16.7 \%(5 \mu \mathrm{g} / \mathrm{ml}$ LPS) and 26.3\% (10 $\mu \mathrm{g} / \mathrm{ml}$ LPS) (Fig. 1B and C). The release of IL-1 $\beta$, IL- 6 and TNF- $\alpha$ was gradually upregulated by LPS in a concentration-dependent manner (Fig. 1D-F). Taken together, these results indicated that LPS reduced cell viability, but promoted apoptosis and pro-inflammatory factor secretion in chondrocytes in a dose-dependent manner, suggesting that LPS may mimic the inflammatory injury of OA in knee articular chondrocytes in vitro. Moreover, OA chondrocytes isolated from patients with $\mathrm{OA}$ also exhibited increased apoptosis and inflammatory response compared with control chondrocytes (Fig. S3A-E).

miR-200b expression is downregulated in OA cartilage and LPS-treated chondrocytes. miR-200b expression was detected using RT-qPCR, and it was revealed that the level of miR-200b was lower in cartilage samples isolated from patients with OA compared with that in normal patient samples (Fig. 2A). Consistently, LPS treatment altered miR-200b expression level in chondrocytes, as 5 and $10 \mu \mathrm{g} / \mathrm{ml}$ LPS resulted in a significant decrease in miR-200b level compared with that in the blank group ( $0 \mu \mathrm{g} / \mathrm{ml}$ LPS; Fig. 2B). These data indicated that miR-200b expression was downregulated in patients with OA and LPS-stimulated chondrocytes.
FUT4 expression is directly modulated by miR-200b. Recently, FUT4 has been reported to be aberrantly upregulated in OA cartilage tissues (19), therefore we hypothesized that FUT4 may serve an important role in knee articular chondrocytes. FUT4 was predicted by TargetScan to be a potential target of miR-200b, and the potential binding sites are presented in Fig. 3A. To examine this hypothesis, dual-luciferase reporter assay and RIP assay were subsequently conducted in miRNA mimic-transfected $293 \mathrm{~T}$ cells and primary chondrocytes, respectively. Transfection of miR-200b mimic resulted in overexpression of miR-200b in 293T cells (Fig. S4A), which significantly reduced the luciferase activity of FUT4-WT, but not that of FUT4-MUT compared with miR-NC (Fig. 3B). In chondrocytes, miR-200b mimic also induced higher expression levels of miR-200b compared with miR-NC (Fig. S4B), which resulted in an enrichment of FUT4 mRNA in RIP-Ago2 immunoprecipitated complexes (Fig. 3C). Moreover, following co-transfection of miR-200b mimic with either FUT4-WT or FUT4-MUT, miR-200b expression was highly induced, and FUT4 mRNA expression level was reduced compared with miR-NC-transfected cells (Fig. S4C and D). Therefore, these results suggested a direct binding relationship between miR-200b and FUT4.

FUT4 is upregulated in injured cartilage tissues and chondrocytes, and modulated by miR-200b. Subsequently, the expression of FUT4 was examined using both RT-qPCR and western blotting. The expression of FUT4 at the mRNA (Fig. 4A) and protein level (Fig. 4B) was upregulated in patients with OA compared with control patients, as well as in LPS-treated chondrocytes compared with control cells (Fig. 4C and D). Moreover, overexpression of miR-200b via mimic transfection reduced and knockdown of miR-200b via anti-miR-200b transfection increased the FUT4 protein expression level in chondrocytes (Fig. 4E and F, respectively). The transfection efficiency of miR-200b mimic and anti-miR-200b is presented in Fig. S4B. These results demonstrated that FUT4 was highly expressed in injured cartilage tissues and chondrocytes and it was negatively modulated by miR-200b.

Restoring FUT4 levels partially reverses the miR-200b-induced protective effect in LPS-induced inflammatory injury in chondrocytes in vitro. The roles of miR-200b and FUT4 in the LPS-induced OA model in chondrocytes were explored. The pcDNA-FUT4 vector was used to overexpress FUT4 in chondrocytes, and chondrocytes were transfected with miR-200b mimic alone or together with pcDNA-FUT4 vector or empty vector. FUT4 mRNA level was significantly upregulated in cells transfected with pcDNA-FUT4 compared with those transfected with the empty vector (Fig. S4E). The downregulation of FUT4 induced by miR-200b overexpression was attenuated by transfection with pcDNA-FUT4 (Fig. 5A). After cell treatment with $10 \mu \mathrm{g} / \mathrm{ml}$ LPS for $48 \mathrm{~h}$, the apoptosis rate was decreased from 30.5 to $16.9 \%$ in chondrocytes transfected with miR-200b mimic compared with those transfected with miR-NC, while it was increased to $22.8 \%$ in cells transfected with pcDNA-FUT4 (Fig. 5B). Moreover, the high level of IL-1 $\beta$, IL-6 and TNF- $\alpha$ in LPS-treated chondrocytes was decreased by miR-200b mimic transfection, while this suppression was reversed following FUT4 overexpression (Fig. 5C-E). These 
A

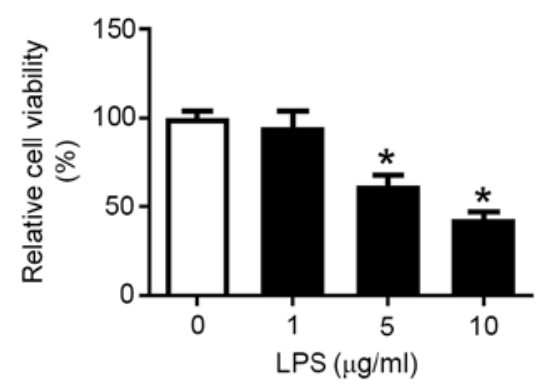

c

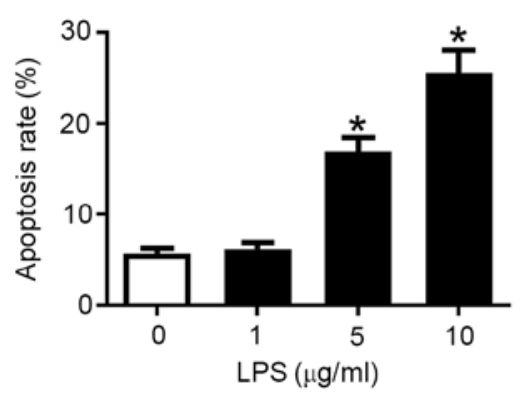

B
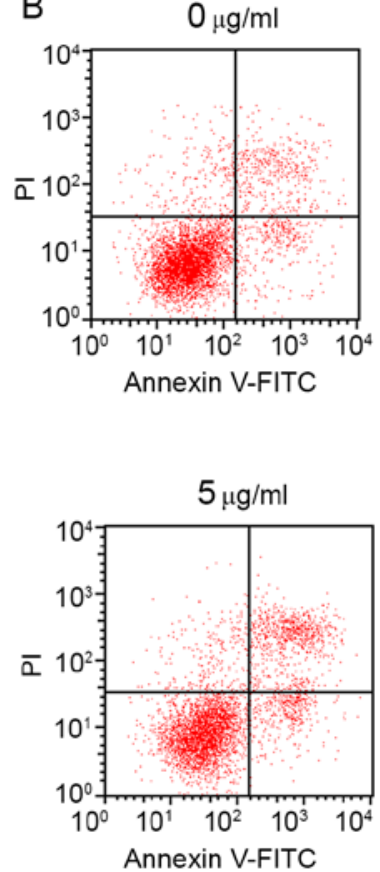
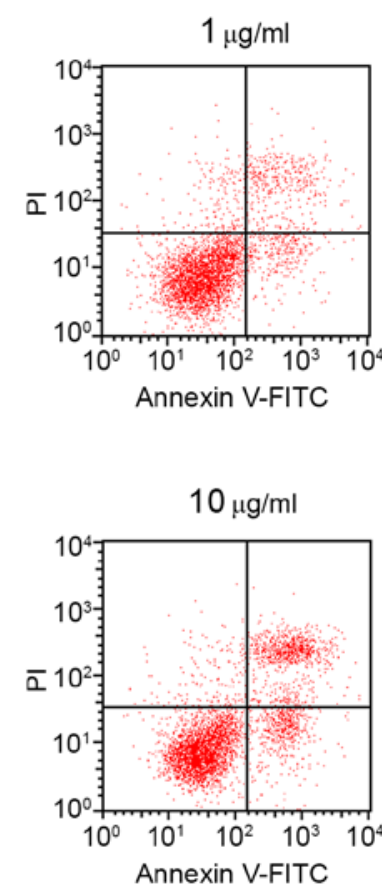

D

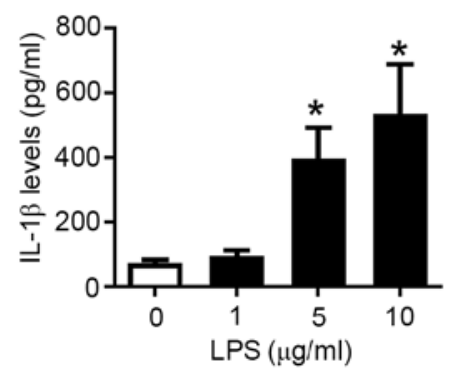

E

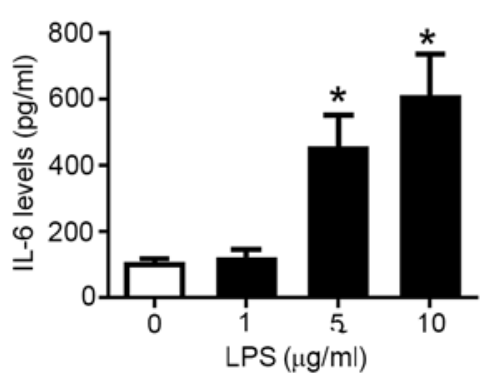

F

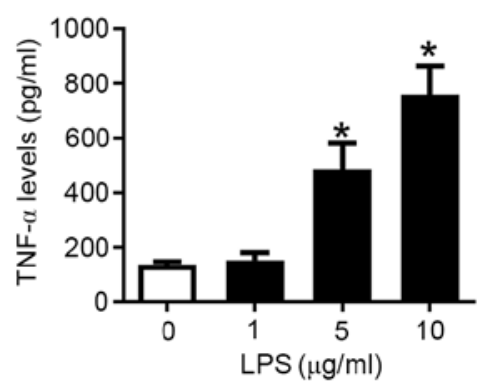

Figure 1. Inflammatory injury induced by LPS in knee articular chondrocytes. Knee articular chondrocytes were isolated from normal cartilage tissues, and subsequently exposed to LPS $(0,1,5$ and $10 \mu \mathrm{g} / \mathrm{ml})$. (A) Cell viability was measured using Cell Counting Kit- 8 assay. (B) Apoptosis was detected by flow cytometry and (C) apoptosis rate was determined. The production of (D) IL-1 $\beta$, (E) IL-6 and (F) TNF- $\alpha$ was evaluated by ELISA. ${ }^{*}<0.05$ vs. $0 \mu$ g/ml LPS. LPS, lipopolysaccharide.

data demonstrated that miR-200b protected chondrocytes from LPS-induced inflammatory injury in vitro by inhibiting FUT4.

\section{Discussion}

$\mathrm{OA}$ is a degenerative joint disease and its effective treatments primarily include the relief of pain and inflammation (28). One key pathological feature of OA is articular cartilage degeneration, which is largely attributed to the apoptosis of chondrocytes (29). Statistics have revealed that $\sim 10 \%$ of the population suffer from OA, especially the elderly (30). At present, the available treatments are often accompanied by severe side effects. Therefore, there is a requirement to develop therapies for OA by identifying novel target genes using patients with OA tissues and cells, as well as multiple stimuli-induced OA models. In the present study, chondrocytes were isolated from healthy patients and patients with OA, and normal chondrocytes were subjected to LPS stimulation.

LPS-induced inflammatory injury in chondrocytes has been widely studied $(23,25)$. LPS has been reported to result in multiple organ damage by stimulating pro-inflammatory cytokine secretion and inflammation-related signaling pathways (23). For example, Jia et al (4) revealed that LPS stimulation effectively attenuated cell viability, and increased apoptosis and the production of IL-1 $\beta$, IL- 6 and TNF- $\alpha$ in knee articular chondrocytes ex vivo compared with LPS-untreated chondrocytes. Emerging evidence has indicated the involvement of non-coding RNAs, including miRNAs and long non-coding RNAs in ECM degeneration and chondrocyte reduction in LPS-stimulated chondrogenic ATDC5 cells $(31,32)$. For instance, overexpression of miR-125b was revealed to inhibit the LPS-induced inflammatory injury by suppressing MIP-1 $\alpha$ expression and $\mathrm{NF}-\kappa \mathrm{B}$ and JNK signaling activation (4). On the contrary, high expression of miR-146a has been indicated to aggravate the LPS-induced inflammatory injury by downregulating CXCR4 and inhibiting the PI3K/AKT and Wnt/ $\beta$-catenin signaling pathways (24). In the present study, the expression levels and the role of miR-200b in an OA model of inflammatory injury were investigated. The results indicated that miR-200b was downregulated in OA cartilage tissues and LPS-stimulated chondrocytes, which was in accordance with 

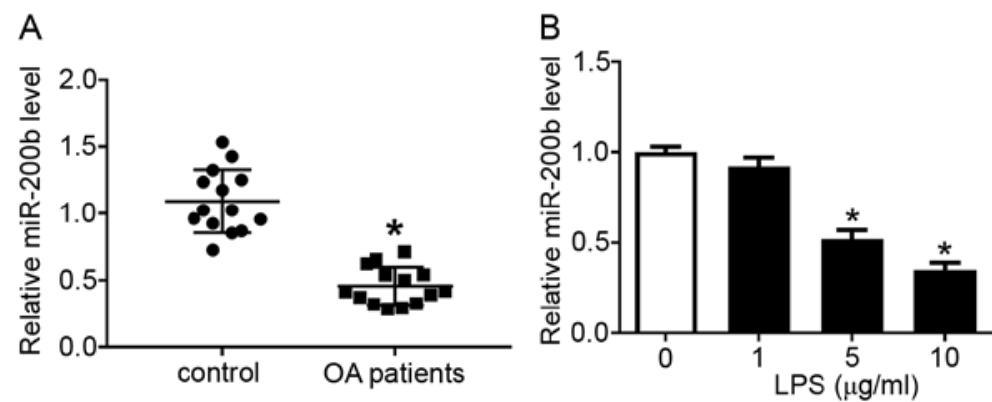

Figure 2. miR-200b expression is downregulated in patients with OA and LPS-induced chondrocytes. (A) Expression level of miR-200b in cartilage samples isolated from normal control patients $(n=14)$ and patients with OA $(n=14)$. ${ }^{*} \mathrm{P}<0.05$ vs. control. (B) Expression level of miR-200b in LPS-treated normal chondrocytes isolated from control patients. "P<0.05 vs. $0 \mu \mathrm{g} / \mathrm{ml}$ LPS. LPS, lipopolysaccharide; miR-200b, microRNA-200b-3p; OA, osteoarthritis.

A

Position 741-747 of FUT4 3'UTR

FUT4-WT 5'-UGACUUAGgGGAGUUCAGUAUUU-3'

miR-200b 3'-AGUAGUAAUGGUCCGUCAUAAU-5

FUT4-MUT 5'-UGACUUAGGGGAGUACUGGGCU-3'
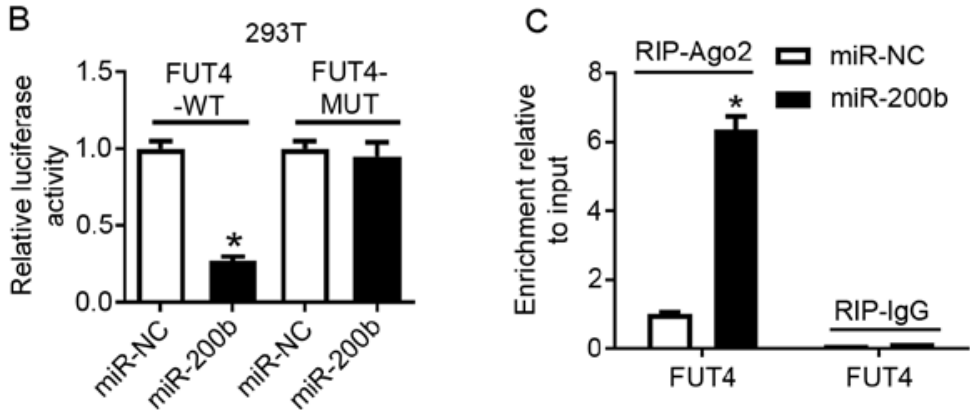

Figure 3. FUT4 is negatively regulated by miR-200b via direct binding. (A) The putative binding sites of miR-200b in FUT4 3'-UTR were predicted, and luciferase reporter vectors containing the FUT4-WT or FUT4-MUT were constructed. (B) Luciferase assay in 293T cells co-transfected with FUT4-WT or FUT4-MUT and miR-200b mimic or miR-NC. (C) Reverse transcription-quantitative PCR following RIP assay was used to detect the mRNA expression level of FUT4 in RIP-Ago2 and IgG-RIP-IgG in chondrocytes transfected with miR-200b or miR-NC. "P<0.05 vs. miR-NC. miR-200b, microRNA-200b-3p; FUT4, fucosyltransferase 4; NC, negative control; RIP, RNA immunoprecipitation; WT, wild-type, MUT, mutant; UTR, untranslated region; Ago2, argonaute-2 .

the finding of Wu et al (16). They proposed that miR-200b might function as a repair factor for OA cartilage, because its upregulation suppressed the degradation of ECM in OA cartilage cells ex vivo, as indicated by the lower MMP and higher collagen II levels in miR-200b mimic-transfected cells compared with negative control-transfected cells (16). Similarly, the present study demonstrated a suppressive role of miR-200b in apoptosis and the inflammatory response in LPS-treated chondrocytes in vitro. However, the role of miR-200b in ECM degradation requires further investigation.

miR-200b is considered to function as a regulatory factor of cell proliferation and motility in a number of types of cancer. For example, the epithelial-mesenchymal transition and tumor growth of glioma were suppressed by miR-200b overexpression via downregulating ERK5 expression (33). Moreover, miR-200b downregulation by low p73 has been indicated to promote androgen independence in prostate cancer cells (34). Nevertheless, few studies have investigated the potential functions of miR-200b in OA pathogenesis. Additional evidence is required to verify the role of miR-200b in vivo.
In the present study, overexpression of FUT4 was revealed to inhibit the miR-200-mediated protective effect in knee articular chondrocyte injury in vitro. DNA (cytosine-5)-methyltransferase 3A and FUT4 have been identified as downstream target genes of miR-200b (16). FUT4 belongs to the fucosyltransferase family, which is a group of fucosylation synthases (21). It has been reported that FUTs are associated with signal transduction, inflammation, tumor progression and metastasis. For example, FUTs have been reported to mediate multidrug resistance in human hepatocellular carcinoma via the PI3K/AKT signaling pathway (18). In arthritis, FUT1 and FUT7 were upregulated in RA synovial fibroblast cells and fluid, respectively $(19,20)$. FUT1 and FUT2 have been demonstrated to mediate angiogenesis and inflammatory cell adhesion in RA. However, to the best of our knowledge, FUTs in OA have not been widely studied with the exception of one study. According to the results of $\mathrm{Hu}$ et al (21), the expression profile of FUT genes in healthy and OA human cartilage tissues was unraveled, and the expression level of FUT4, FUT1, FUT2 and FUT3 was notably increased in OA tissues. According 


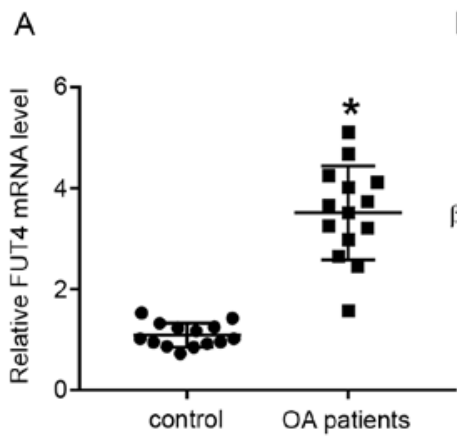

D

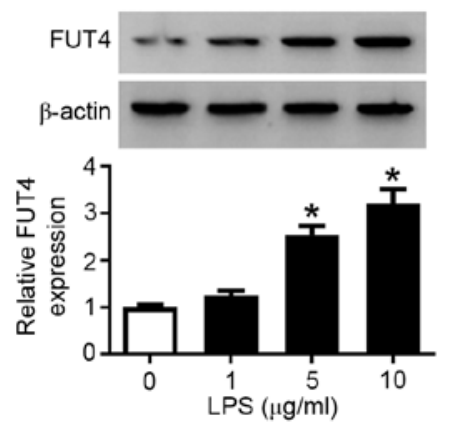

B

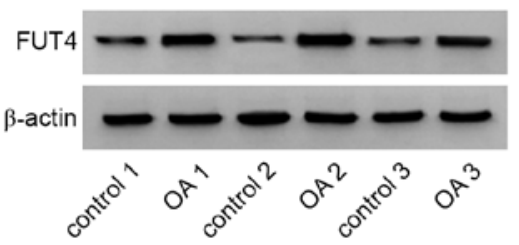

C

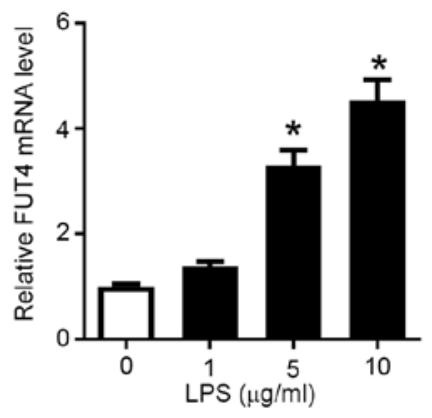

F

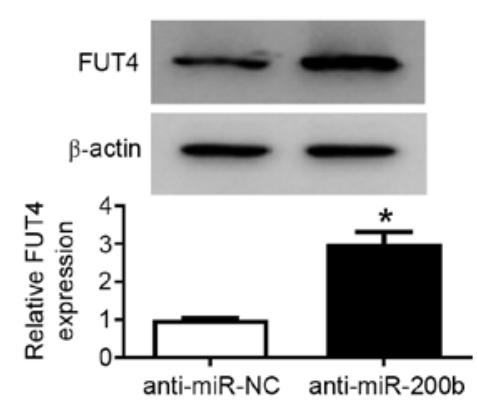

Figure 4. Expression of FUT4 in OA tissues and LPS-induced chondrocytes. (A) FUT4 mRNA and (B) protein level in cartilage samples was detected by RT-qPCR and western blotting. ${ }^{*} \mathrm{P}<0.05$ vs. control tissues. (C) RT-qPCR and (D) western blotting was used to measure FUT4 levels in LPS-stimulated chondrocytes isolated from normal cartilages. ${ }^{*} \mathrm{P}<0.05 \mathrm{vs} .0 \mu \mathrm{g} / \mathrm{ml}$ LPS. Western blotting was used to examine FUT4 expression in normal chondrocytes transfected with (E) miR-NC or miR-200b and (F) anti-miR-NC or anti-miR-200b. ${ }^{*} \mathrm{P}<0.05$ vs. the respective NC. FUT4, fucosyltransferase 4; NC, negative control; RT-qPCR, reverse transcription-quantitative PCR; OA, osteoarthritis; LPS, lipopolysaccharide; miR-200b, microRNA-200b-3p.

A

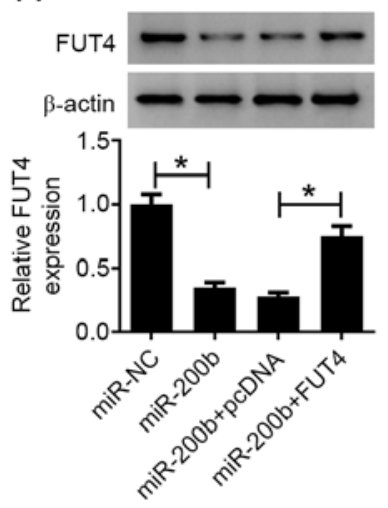

C

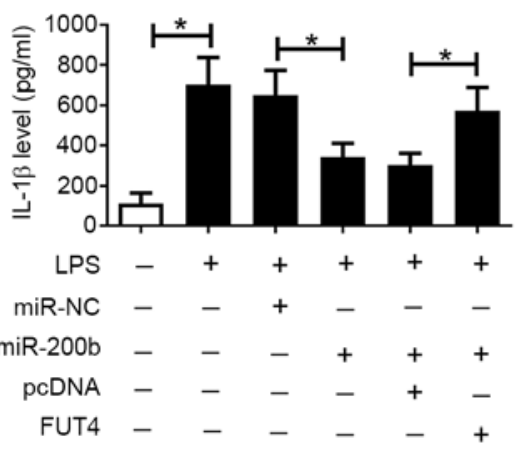

B

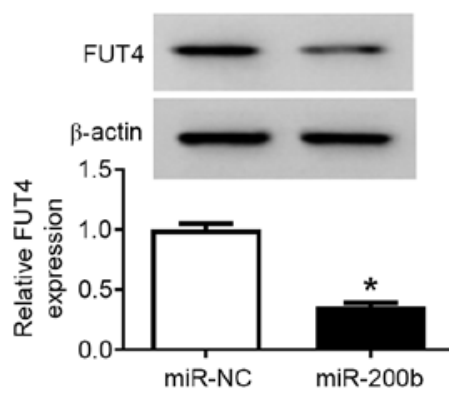


to the findings of the present study, FUT4 expression at the mRNA and protein level was increased in OA cartilage tissues and LPS-treated chondrocytes compared with control tissues and cells, respectively. Functionally, overexpression of FUT4 attenuated the miR-200b-induced suppressive effects on the apoptosis rate and the expression level of IL-1 $\beta$, IL- 6 and TNF- $\alpha$ in LPS-treated chondrocytes.

In consideration of the protective role of miR-200b overexpression in injured chondrocytes, including OA chondrocytes ex vivo (16) and LPS-stimulated chondrocytes in vitro (from the results of the current study), miR-200b may be a cartilage repair gene in OA. The findings of the current study suggested that miR-200b may be a potential candidate to improve chondrocyte viability in knee OA by affecting apoptosis and inflammatory response of injured chondrocytes, as well as ECM degeneration, which is indicated by a previous study (35). In the future, additional studies should be performed to explore the signaling pathways of the miR-200b/FUT4 axis involved in the progression of chondrocyte injury, such as the NF- $\mathrm{B}$ (21) and MAPK/ERK (16) pathway.

Collectively, the present study demonstrated that miR-200b was downregulated in cartilage tissues from patients with OA, and upregulating miR-200b attenuated LPS-induced apoptosis and inflammatory response in knee articular chondrocytes in vitro via targeting FUT4.

\section{Acknowledgements}

Not applicable.

\section{Funding}

No funding was received.

\section{Availability of data and materials}

The datasets used and/or analyzed during the present study are available from the corresponding author on reasonable request.

\section{Authors' contributions}

SW and ZZ conceived the study, designed the methodology and performed the final analysis. YL and SW performed the experiments, data curation, validation and investigation. $\mathrm{ZZ}$ performed GraphPad software analysis. YL wrote the original draft. SW reviewed and edited the manuscript. All authors read and approved the final manuscript.

\section{Ethics approval and consent to participate}

The present study was conducted in accordance with the Declaration of Helsinki and was approved by the Ethics Committee of Yan'an Peoples Hospital (Yan'an, China; February 15, 2019). Written informed consent was obtained from each patient.

\section{Patient consent for publication}

Written informed consent for publication was obtained from each patient.

\section{Competing interests}

The authors declare that they have no competing interests.

\section{References}

1. van den Bosch MHJ: Inflammation in osteoarthritis: Is it time to dampen the alarm(in) in this debilitating disease? Clin Exp Immunol 195: 153-166, 2019.

2. Loeser RF, Collins JA and Diekman BO: Ageing and the pathogenesis of osteoarthritis. Nat Rev Rheumatol 12: 412-420, 2016.

3. Greene MA and Loeser RF: Aging-related inflammation in osteoarthritis. Osteoarthritis Cartilage 23: 1966-1971, 2015.

4. Jia J, Wang J, Zhang J, Cui M, Sun X, Li Q and Zhao B: MiR-125b inhibits LPS-induced inflammatory injury via targeting MIP-1 $\alpha$ in chondrogenic cell ATDC5. Cell Physiol Biochem 45: 2305-2316, 2018

5. Kourtis A, Adamopoulos PG, Papalois A, Iliopoulos DC, Babis GC and Scorilas A: Quantitative analysis and study of the mRNA expression levels of apoptotic genes BCL2, BAX and BCL2L12 in the articular cartilage of an animal model of osteoarthritis. Ann Transl Med 6: 243, 2018.

6. Chen Y, Sun Y, Pan X, Ho K and Li G: Joint distraction attenuates osteoarthritis by reducing secondary inflammation, cartilage degeneration and subchondral bone aberrant change. Osteoarthritis Cartilage 23: 1728-1735, 2015.

7. Cong L, Zhu Y and Tu G: A bioinformatic analysis of microRNAs role in osteoarthritis. Osteoarthritis Cartilage 25: 1362-1371, 2017.

8. Jeffries MA, Donica M, Baker LW, Stevenson ME, Annan AC, Humphrey MB, James JA and Sawalha AH: Genome-wide DNA methylation study identifies significant epigenomic changes in osteoarthritic cartilage. Arthritis Rheumatol 66: 2804-2815, 2014.

9. Li B, Bai L, Shen P, Sun Y, Chen Z and Wen Y: Identification of differentially expressed microRNAs in knee anterior cruciate ligament tissues surgically removed from patients with osteoarthritis. Int J Mol Med 40: 1105-1113, 2017.

10. Song J, Ahn C, Chun CH and Jin EJ: A long non-coding RNA, GAS5, plays a critical role in the regulation of miR-21 during osteoarthritis. J Orthop Res 32: 1628-1635, 2014.

11. Yang R, Zhang D, Yu K, Sun L, Yang J, Zhao C, Li X and Chen Y: Detection of miR-22, miR-140 and bone morphogenetic proteins (BMP)-2 expression levels in synovial fluid of osteoarthritis patients before and after arthroscopic debridement. Med Sci Monit 24: 863-868, 2018.

12. Zhang HX, Sun C, Yu HC, Song B and Pan ZX: Targeted inhibition of $\beta$-catenin by miR-320 and decreased MMP-13 expression in suppressing chondrocyte collagen degradation. Eur Rev Med Pharmacol Sci 22: 5828-5835, 2018.

13. Feng B, Wang R and Chen L: Review of miR-200b and cancer chemosensitivity. Biomed Pharmacother 66: 397-402, 2012.

14. Zeng F, Xue M, Xiao T, Li Y, Xiao S, Jiang B and Ren C: MiR-200b promotes the cell proliferation and metastasis of cervical cancer by inhibiting FOXG1. Biomed Pharmacother 79: 294-301, 2016.

15. Zheng Q, Cui X, Zhang D, Yang Y, Yan X, Liu M, Niang B, Aziz F, Liu S, Yan Q and Liu J: miR-200b inhibits proliferation and metastasis of breast cancer by targeting fucosyltransferase IV and $\alpha 1,3$-fucosylated glycans. Oncogenesis 6: e358, 2017.

16. Wu J, Tao Y, Shang A, Wang W, Zhang Y, Hu L, Wang J, Wang Y and Guo N: Effect of the interaction between MiR-200b-3p and DNMT3A on cartilage cells of osteoarthritis patients. J Cell Mol Med 21: 2308-2316, 2017.

17. Keeley TS, Yang S and Lau E: The diverse contributions of fucose linkages in cancer. Cancers (Basel) 11: 1241, 2019.

18. Cheng L, Luo S, Jin C, Ma H, Zhou H and Jia L: FUT family mediates the multidrug resistance of human hepatocellular carcinoma via the PI3K/Akt signaling pathway. Cell Death Dis 4: e923, 2013.

19. De Benedetti F, Pignatti P, Biffi M, Bono E, Wahid S, Ingegnoli F, Chang SY, Alexander H, Massa M, Pistorio A, et al: Increased expression of alpha(1,3)-fucosyltransferase-VII and P-selectin binding of synovial fluid $\mathrm{T}$ cells in juvenile idiopathic arthritis. J Rheumatol 30: 1611-1615, 2003.

20. Isozaki T, Ruth JH, Amin MA, Campbell PL, Tsou PS, Ha CM, Haines GK, Edhayan G and Koch AE: Fucosyltransferase 1 mediates angiogenesis, cell adhesion and rheumatoid arthritis synovial tissue fibroblast proliferation. Arthritis Res Ther 16: R28, 2014. 
21. Hu J, Wang Z, Pan Y, Ma J, Miao X, Qi X, Zhou H and Jia L: MiR-26a and miR-26b mediate osteoarthritis progression by targeting FUT4 via NF- $\kappa$ B signaling pathway. Int J Biochem Cell Biol 94: 79-88, 2018.

22. Chaby R: Lipopolysaccharide-binding molecules: Transporters, blockers and sensors. Cell Mol Life Sci 61: 1697-1713, 2004.

23. Huang ZY, Stabler T, Pei FX and Kraus VB: Both systemic and local lipopolysaccharide (LPS) burden are associated with knee OA severity and inflammation. Osteoarthritis Cartilage 24: $1769-1775,2016$

24. Sun T, Li X, Song H, Gao F, Zhou G, Li X, Chen Z and Chen L: MiR-146a aggravates LPS-induced inflammatory injury by targeting CXCR4 in the articular chondrocytes. Cell Physiol Biochem 44: 1282-1294, 2017.

25. Wang Y and Kong D: MicroRNA-136 promotes lipopolysaccharide-induced ATDC5 cell injury and inflammatory cytokine expression by targeting myeloid cell leukemia 1 . J Cell Biochem 119: 9316-9326, 2018.

26. Hubertsson J, Petersson IF, Thorstensson CA and Englund M: Risk of sick leave and disability pension in working-age women and men with knee osteoarthritis. Ann Rheum Dis 72: 401-405, 2013.

27. Livak KJ and Schmittgen TD: Analysis of relative gene expression data using real-time quantitative PCR and the 2(-Delta Delta C(T)) method. Methods 25: 402-408, 2001

28. van Middelkoop M, Arden NK, Atchia I, Birrell F, Chao J, Rezende MU, Lambert RG, Ravaud P, Bijlsma JW, Doherty M, et al: The OA Trial Bank: meta-analysis of individual patient data from knee and hip osteoarthritis trials show that patients with severe pain exhibit greater benefit from intra-articular glucocorticoids. Osteoarthritis Cartilage 24 $1143-1152,2016$
29. Hwang HS and Kim HA: Chondrocyte apoptosis in the pathogenesis of osteoarthritis. Int J Mol Sci 16: 26035-2654, 2015.

30. Feldmann M: Pathogenesis of arthritis: Recent research progress. Nat Immunol 2: 771-773, 2001.

31. Li F, Sun J, Huang S, Su G and Pi G: LncRNA GAS5 overexpression reverses LPS-induced inflammatory injury and apoptosis through up-regulating KLF2 expression in ATDC5 chondrocytes. Cell Physiol Biochem 45: 1241-1251, 2018.

32. Pan L, Liu D, Zhao L, Wang L, Xin M and Li X: Long noncoding RNA MALAT1 alleviates lipopolysaccharide-induced inflammatory injury by upregulating microRNA-19b in murine chondrogenic ATDC5 cells. J Cell Biochem 119: 10165-10175, 2018.

33. Wu J, Cui H, Zhu Z and Wang L: MicroRNA-200b-3p suppresses epithelial-mesenchymal transition and inhibits tumor growth of glioma through down-regulation of ERK5. Biochem Biophys Res Commun 478: 1158-1164, 2016.

34. He M, Liu Y, Deng X, Qi S, Sun X, Liu G, Liu Y, Liu Y and Zhao M: Down-regulation of miR-200b-3p by low p73 contributes to the androgen-independence of prostate cancer cells. Prostate 73: 1048-1056, 2013.

35. Gwam CU, Etcheson JI, George NE, Mistry JB, Mohamed N, Patel A, Gwam PN, Piuzzi NS and Delanois RE: Presentation of knee osteoarthritis in the emergency department: A problem worth mentioning? Surg Technol Int 31: 277-284, 2017.

This work is licensed under a Creative Commons Attribution-NonCommercial-NoDerivatives 4.0 International (CC BY-NC-ND 4.0) License. 